\title{
The Brunner-Lei-Peters Resilience Scale
}

\author{
Lexi Brunner \\ Department of Psychology, University of Alberta, Edmonton, Alberta \\ Corresponding author: Ibrunner@ualberta.ca
}

\section{ABSTRACT}

Resilience is an important attribute for mental health. Positive benefits felt by resilient individuals include fewer depressive episodes, and better coping strategies. Due to the positive outcomes experienced by resilient people, it may be clinically useful to examine this construct within a psychotherapeutic context. Accordingly, we created the Brunner-Lei-Peters Resilience Scale, which included a preliminary test of 70 items and 10 validity items; we tested the scale on a sample of 150 people. Participants were representative of the general population with about an equal representation of gender and a wide age range from 18 to 66 years of age. Although the scale initially included 70 items, through psychometric analysis, we reduced the scale to 15 items, while maintaining sound psychometric validity and reliability. Clinical implications and limitations of the scale are discussed.

\section{Introduction}

Being able to cope with stressors is an important predictor for successful psychological wellbeing. For example, adults with effective coping strategies to stress are found to also have greater psychological well-being (Mayordomo, Viguer, Sales, Satorres, \& Melendez, 2016). As well, higher levels of resilience in older adults are associated with lower depressive symptoms (Wermelinger, Lucchetti, \& Lucchetti, 2017). In essence, the capacity to be resilient seems to provide a buffer from even extreme stressors. For the purposes of the study, we viewed resilience as the ability to bounce back from adversity. As the capacity to be resilient is an abstract concept, we operationalized resilience into clear questions designed to tap into this capacity (see Appendix A).

According to a recent methodological review of nineteen resilience scales, the Brief Resilience Scale scores best overall in psychometric rigor (Windle, Bennett, \& Noyes, 2011). Despite this, the Brief Resilience Scale is limited in its validation by its overreliance on an older female sample, and by its failure to assess broader areas of resilience (e.g., availability of support and resources). Per Windle, Bennett, and Noyes' (2011) recommendations, we

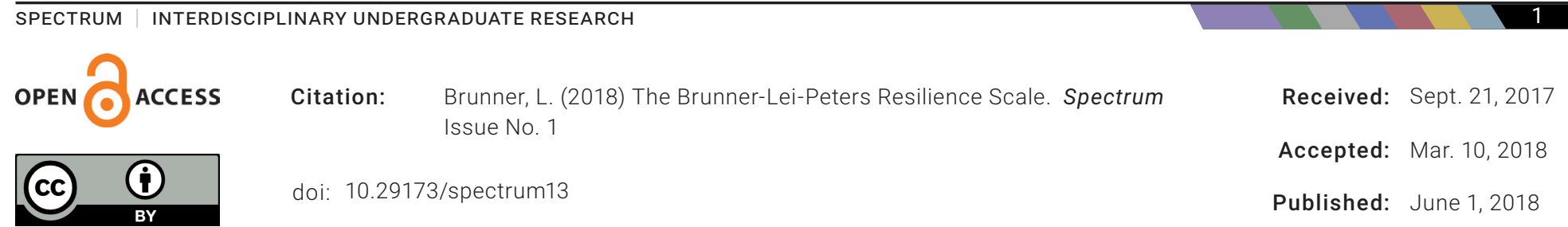


constructed our scale to be validated over a wide range of ages, be reasonably gender equivalent, and tap into more components of resilience.

As the current research only sampled adults $(18+)$, we created resilience items geared towards assessment of an adult sample. Based on Taormina's (2015) insights, we included four subcomponents of resilience in adults: determination, endurance, adaptability and recuperability. Hence, many of our items assessed these four main dimensions (e.g., determination: "I am determined to succeed"; endurance: "I am empowered to succeed even in the presence of adverse circumstances"; adaptability: "I am adaptable"; and recuperability: "I recover from failures"). Although these four dimensions encompass a portion of resilience, we also hypothesized other components of resilience. One element may be an ability to find humor in otherwise negative and serious situations. Kuiper (2012) uncovered that humor may help individuals as it leads to a high level of positive affect, which aids successful coping with trauma. In accordance with this research, we incorporated a few resilience items that asked participants about humor (e.g., "I can laugh at myself"). Another related component in resilience is the ability to utilize resources effectively to manage life's stressors. Prior research found that social and personal resources aided chronically-ill youth in coping with their adverse health; those without these resources were at risk for depression and a lower quality of life (Oleś, 2015). Thus, we also incorporated items that seemed to address participants' usage of available resources: "I am resourceful."

\section{Methods}

\section{Participants}

Participant recruitment occurred over a period of two weeks via Google Forms. Survey invitations were sent out to colleagues, friends, family, and other students. In total, 150 individuals completed the survey. Ethnic breakdown is as follows: over half of the sample was Caucasian (66.7\%); a substantial percentage of participants were Asian/Pacific Islander (31.3\%); and the remainder of participants were Native American/Aboriginal (4.7\%); Hispanic/Latino (2\%); African American $(0.7 \%)$ and Other (2\%). Our percentages pooled to over $100 \%$ as we directed participants to choose all ethnic identities that applied to them. Ages ranged from 18 years to 66 years $(M=28.2, S D=$ 11.47). The mean age was right-skewed by a few older participants so we calculated the median age which was 23 . This age better represents our data as the mode of participants (24.5\%) were 21 years of age. Sixty percent of participants were assigned female sex at birth and $40 \%$ were male. Gender identity for the sample was consistent, with $40 \%$ indicating their male gender identity and the rest citing female as their gender identity.

\section{Materials}

All three researchers independently formulated questions and then deleted duplicate items at a question-formation meeting. As some resilience scales already exist, we re-worded six items from two reputable resilience scales found online (Duckworth, Peterson, Matthews, \& Kelly 2007; Smith, Dalen, Wiggins, Tooley, Christopher, \& Bernard, 2008). In total, there were 80 items proposed, 10 of which were purposefully chosen as validity items. Although the 10 validity questions were not cross-validated, we used our psychometric judgment to compose high face-validity questions that directly assessed the resilience construct (e.g., "I am resilient). We also reverse-scored 15 items as a means of deterring response biases (social desirability and acquiescence). Likewise, to minimize the effects of response bias, we informed students that their responses were anonymous, and made the title of our questionnaire purposefully ambiguous (Psychometric Questionnaire), rather than stating that the purpose was to study resilience. All 70 items plus the 10 validity items are included in 
Appendix A, and the 15 surviving items are in Appendix $B$. Although the validity items are shown separately in the appendices, when administering the questionnaire, all the validity items were randomly mixed with the other 70 items.

\section{Procedure}

Participants rated on a five-point scale how strongly they agreed/disagreed with each item from 1 (strongly disagree) to 5 (strongly agree). Total scores were computed in Microsoft Excel for each participant. Validity score items were computed independently from the total scores of the scale. Each item's total score was separately correlated to the total validity score. By doing so, we eliminated items that did not correlate strongly to the total validity score measure (items with correlations less than 0.53). Our most strongly correlated items kept in the scale ranged from $r=0.53$ "I have gotten stronger over time" to $r=0.64$ "Even if I fail now, I can do better in the future." Before removing any items and after we reduced the scale to 15 items, we ran tests of reliability, validity, and conducted factor analysis using the Statistical Program for the Social Sciences (SPSS Version 23).

\section{Results}

Through item analysis, the Brunner-Lei-Peters Resilience Scale was reduced from 70 items to 15 items, while maintaining strong validity and reliability in the scale. We removed redundancies to keep the scale psychometrically valid and reliable. Before item removal, $\left(r_{s}=0.86 ; a=0.95\right)$, and after removing the poor items, we still retained strong reliability and validity $\left(r_{s}=0.86 ; a=0.91\right)$. The average score of participants was 58.78, the mode was 56 , and the median score was 59 . As our scale ranged from 1 (strongly disagree) to 5 (strongly agree), a higher score on the measure indicated more reported resilience (after accounting for the reverse-scored items). The maximum score one could attain on the Brunner-Lei-Peters Resilience
Scale was 75, and the lowest score possible was 15. The standard error of measurement for our scale was quite sensitive at 2.78 . As we collected demographic information from all participants, we also chose to examine whether different sexes, ethnicities, or age mattered in total resilience scores. A $t$-test revealed that there were no statistically significant differences between the resilience of males and females: $t$ $(148)=-0.65, p>0.95$, indicating that males $(M=$ $59.38, S D=9.14)$ were similarly resilient to females $(M=58.37 ; S D=9.40)$. Another $t$-test indicated there was no significant difference in resilience between younger ( $<23$ years of age, $M=57.89$, $S D=10.41)$, and older (>23 years of age, $M=$ $60.05, S D=7.72)$ participants $(t(145)=1.32, p<$ 0.30 . It is important to note that our median age of participants was 23, and age differences may be more likely if we had more participants of an older age. Finally, we also computed a One-Way ANOVA to test resilience between ethnic groups: $F$ $(4,132)=0.27, p>0.89$, which was inconclusive.

Reliability and Validity

Inter-item reliabilities of the 15 items utilizing Cronbach's alpha indicate strong internal consistency: $a=0.91$. Our final analysis of items revealed no questions correlated to one another greater than 0.90 , thus each question stood on its own and added separately to the variance. Our items also have strong face validity as on the surface they appear to assess resilience. Upon testing these items, construct validity was ascertained as $r_{s}=0.86$. We utilized Spearman's rank order correlation as our scale was rankordered and had equal intervals between rankings.

\section{Factor Analysis and the Scree Plot}

Factor analysis of the remaining 15 items revealed three factors. However, factor one accounted for $44.40 \%$ of the total variance, so we reduced our data to one factor. The other two factors accounted for negligible variance: Factor $2=7.83 \%$; Factor 3 
$=6.74 \%$. As seen from the scree plot (Figure 1), much of the variance is accounted for by the first component, because the eigenvalue is much larger than the eigenvalues of subsequent components. Thus, as is visible from the scree plot, the amount of variance accounted for by these factors is minimal in comparison and excluded from our analyses. Moreover, all fifteen items loaded onto the first factor ranging from 0.60 ("I have gotten stronger over time") to 0.72 ("I am proactive in tackling challenges"). If we included the additional two factors, it would likely result in more noise and introduce redundancies into the data set as factor 1 accounted for the majority of the variance and all 15 items loaded onto the primary factor.

\section{Cut Off Points}

As seen through Figure 2, the line of best fit explains $79 \%$ of the total variance. We set our base rate score to $40 / 60$ to diagnose only the top $1 / 3$ participants as highly resilient. The lower-bound cutting line was placed at 30 as there appear to be three individuals who are not very resilient and are outliers compared to the rest of the group. The upper bound of the cutting score was placed at 60 out of a maximum of 75. Despite these outliers, most of the data is maximally clumped within a restricted range. We tried to maximize the amount of variability accounted for by placing our cut-off points in optimal locations to minimize the amount of error.

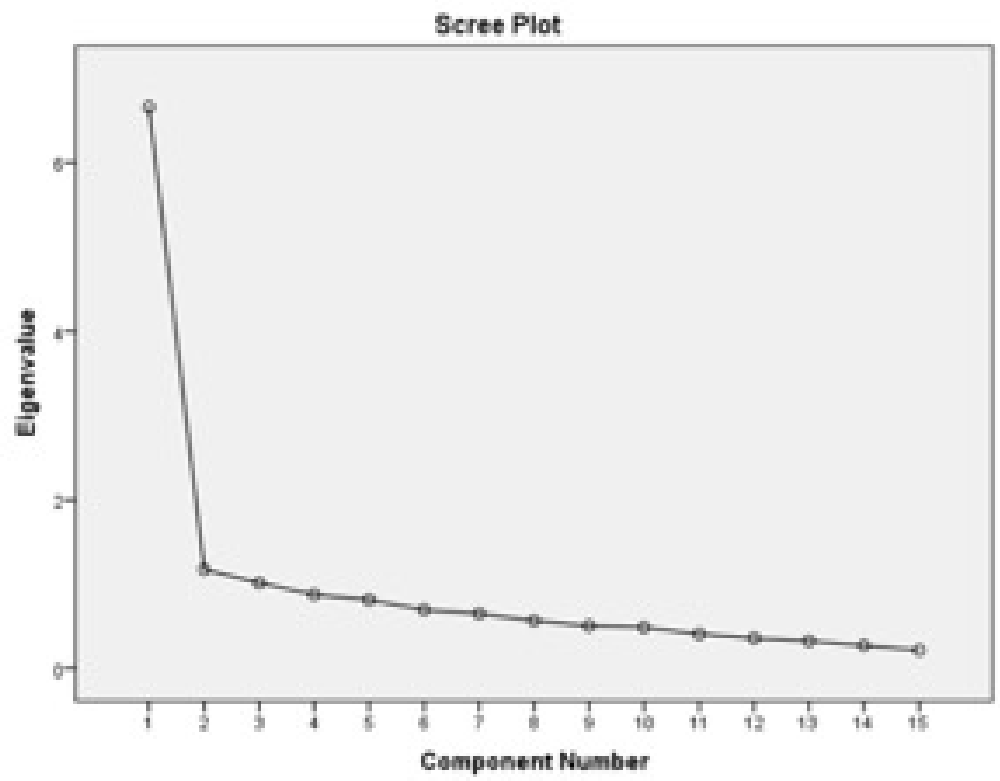

Figure 1: Scree Plot of the 15-items in the Brunner-Lei-Peters Resilience Scale.

\section{Discussion}

Research on resilience is important because the ability to bounce back after adverse situations contributes to positive psychological wellbeing (Mayordomo et al., 2016). For example, Tosun and Dilmac (2015), identified married individuals' levels of resilience were the second most important predictor in conflict resolution style, which is essential for marital success. Research supports that clinical assessment should also focus on dynamic features clients can change rather than just static traits (Rutter, 2013). Accordingly, one study uncovered that resilience may be adaptable (Wilson, 2016). Researchers taught students to practice gratitude as a means of bolstering resilience; those that practiced gratitude increased their ability to be resilient in a challenging educational setting. Although this research is in an educational domain, the study provides support that 


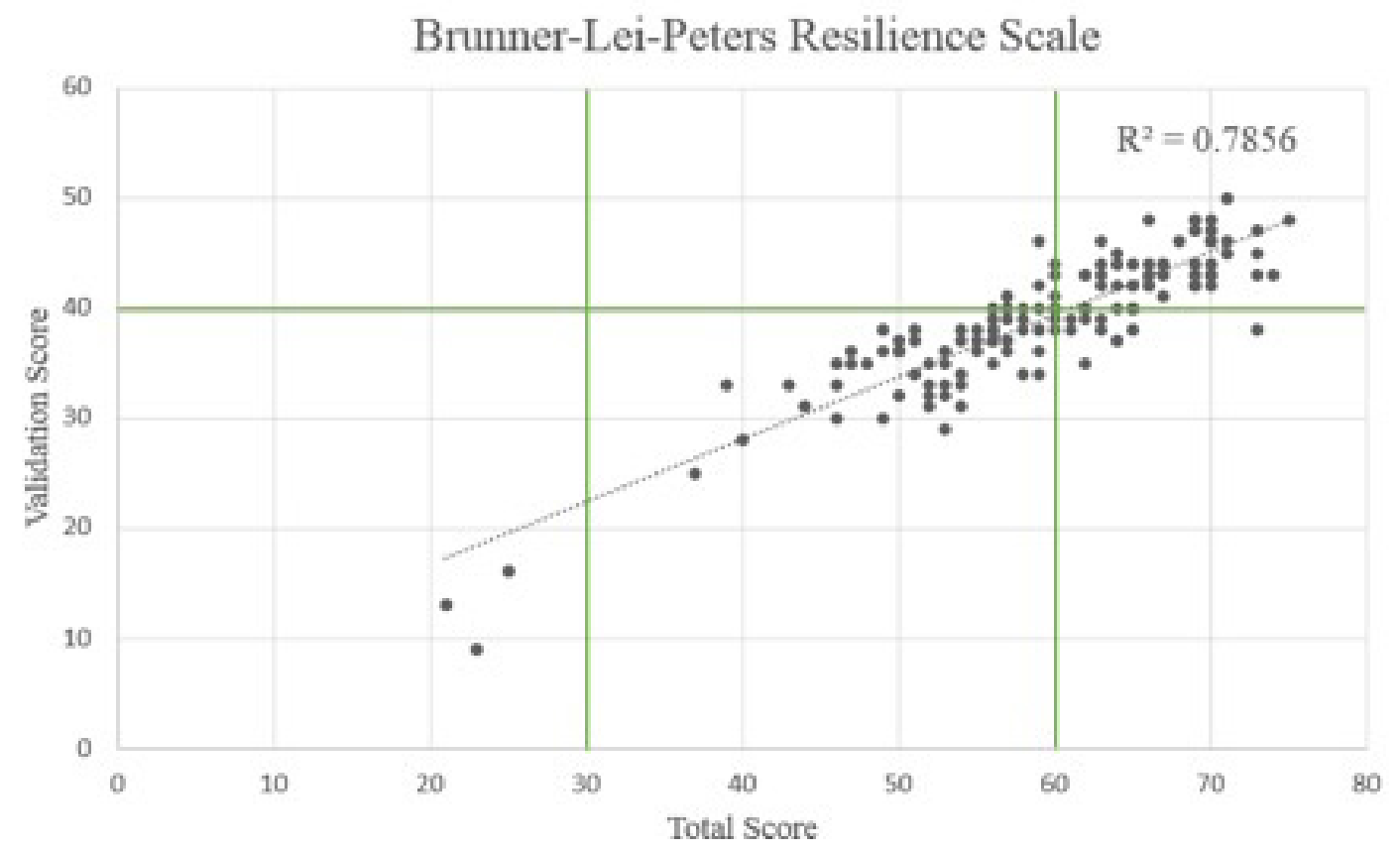

Figure 2: Resilience cutting scores. Base rate score was set to 40 with the two vertical cutting scores at 30 and 60 .

resilience is malleable and thus may be adaptable in therapeutic contexts too. Utilizing a scale akin to ours in a clinical setting may provide an initial baseline assessment of clients' self-perceived resilience. If patients score low on resilience, this provides informative cues about the extent to which they may succeed in psychotherapy.

The purpose of the study was to construct a psychometrically-sound scale of resilience. As resilience is indeed an abstract concept, we formulated items to best address the nomological concept of resilience. Since our questionnaire is short (15 questions), it is easily administered to get a quick view of how resilient clients views themselves. Clinicians may also wish to have others close to the patient fill out the questionnaire and see if there is a disjoint between the clients' perceptions and close others' views. The BrunnerLei-Peters Resilience Scale is general enough to apply to all clients and doesn't focus on specific domains that one may be resilient within (e.g., health, academics, work). Accordingly, in therapy it may be beneficial to use the scale as an initial assessment tool, but also employ prompting questions to ascertain the unique resilient domains of each client. In sum, the Brunner-Lei-Peters Resilience Scale does not act as a substitute for therapy, but rather as a useful starting point.

\section{Future Directions \& Limitations}

Future research may wish to cross-validate and norm the questionnaire on a sub-sample of individuals known to be resilient. Moreover, the sample tested for the current scale relied heavily on convenience sampling - those within the researchers' proximate area who agreed to fill out the questionnaire. Future research would benefit from conducting random sampling as a means of reducing potential biases within the data. The current group of participants who filled out the questionnaire was largely from a college population (via the researchers' social networks) and may differ in some systematic way from the general populace. Despite these limitations, strengths of the questionnaire include its sample size $(N=150)$, relatively diverse age range (18-66), high reliability $(=0.841)$, and reasonably strong validity $\left(r_{s}=0.862\right)$. 


\section{Conclusion}

Overall, the Brunner-Lei-Peters Resilience Scale offers a useful and succinct approach to assess patient resilience in a clinical setting. Although this preliminary study provides promising information to clinicians, more research utilizing the scale is necessary before being able to confidently promote the questionnaire as a psychometric instrument. For example, future research may standardize the scale on a known sample of resilient individuals rather than formulating what the researchers conceive of as valid resilient items. 


\section{References}

Duckworth, A.L., Peterson, C., Matthews, M.D., \& Kelly, D.R. (2007). Grit: Perseverance and passion for long-term goals. Journal of Personality and Social Psychology, 9, 1087-1101. https://doi. org/10.1037/0022-3514.92.6.1087

Kuiper, N. A. (2012). Humor and resiliency: Towards a process model of coping and growth. Europe's Journal of Psychology, 8, 475-491. https://doi.org/10.5964/ejop.v8i3.464

Mayordomo, T., Viguer, P., Sales, A., Satorres, E., \& Melendez, J. C. 2016. Resilience and Coping as Predictors of Well-Being in Adults. The Journal of Psychology: Interdisciplinary and Applied, 150, 809-821. https://doi.org/10.1080/00223980.2016.1203276

Oleś, M. (2015). Resilience and quality of life in chronically ill youth. Health Psychology Report, 3, 220236. https://doi.org/10.5114/hpr.2015.53246

Rutter, M. (2013). Annual Research Review: Resilience - clinical implications. The Journal of Child Psychology and Psychiatry, 54, 474-487. https://doi.org/10.1111/j.1469-7610.2012.02615.x

Smith, B. W., Dalen, J., Wiggins, K., Tooley, E., Christopher, P., \& Bernard, J. (2008). The Brief Resilience Scale: Assessing the ability to bounce back. International Journal of Behavioral Medicine, 15, 194200. https://doi.org/10.1080/10705500802222972

Taormina, R. J. (2015). Adult personal resilience: A new theory, new measure, and practical implications. Psychological Thought, 8, 35-46. https://doi.org/10.5964/psyct.v8i1.126

Tosun, F., \& Dilmac, B. (2015). Predictor relationships between values held by married individuals, resilience, and conflict resolution styles: A model suggestion. Educational Sciences: Theory and Practice, 15, 849-857. https://doi.org/10.12738/estp.2015.4.2588

Wilson, J. T. (2016). Brightening the mind: The impact of practicing gratitude on focus and resilience in learning. Journal of Scholarship of Teaching and Learning, 16, 1-13. https://doi.org/10.14434/ josotl.v16i4.19998

Windle, G., Bennett, K. M., \& Noyes, J. (2011). A methodological review of resilience measurement scales. Health and Quality of Life Outcomes, 9, 1-18. https://doi.org/10.1186/1477-7525-9-8 


\section{Appendix A - Full set of resilience items}

PSYCO 431 Resilience Items

$\begin{array}{ccccc}\text { Strongly Disagree } & \text { Disagree } & \text { Neutral } & \text { Agree } & \text { Strongly Agree } \\ 1 & 2 & 3 & 4 & 5\end{array}$

$(\mathrm{R})$ = reverse-scored

1. I take on challenges even when the outcome is uncertain.

2. I struggle to make it through stressful events $(R)^{\star *}$

3. Obstacles make me stronger.

4. I bounce back quickly after encountering adverse situations.

5. I have gotten stronger over time.

6. I stand up for myself.

7. When faced with difficult situations I cannot overcome them. (R)

8. I face challenges head on.

9. Handling challenges helps me face more difficult situations in the future.

10. It takes me a long time to recover from a stressful event. $(R)$ **

11. I come back even stronger after challenging situations.

12. Resilience is important for success.

13. Obstacles change me for the better.

14. Nothing will hold me back.

15. I am good at finding solutions when problems arise.

16. I help others in need.

17. I cope well with stress.

18. I am empowered to succeed even in the presence of adverse circumstances.

19. Setbacks discourage me. $(\mathrm{R})$ *

20. My troubles in the past have prepared me well for the future.

21. I need to face obstacles head on in order to become a stronger person.

22. Even if I fail now, I can do better in the future.

23. It is hard to be resilient in challenging situations. (R)

24. I have no problem getting through difficult times. **

25. I am resourceful.

26. The future is in my control.

27. When I succeed, it's because I got lucky. (R)

28. I am determined to succeed.

29. I am adaptable.

30. I handle setbacks well.

31. Setbacks are temporary.

32. I am flexible with my plans.

33. I am confident in my abilities.

34. I am a victim of my circumstances. (R)

35. I maintain a hopeful outlook.

36. I have people to count on in difficult situations. 
37. I am comfortable with failure.

38. Even in the hardest circumstances, I manage to find positivity.

39. I take a long time to get over setbacks in life. $(R) \star *$

40. I manage my emotions under stress.

41. I have self-control.

42. When obstacles get in the way of my goals, I become discouraged. (R)

43. I am passionate about my goals.

44. I can handle competing demands.

45. I recover from failures.

46. I am grateful for my adverse experiences.

47. Everything happens for a reason.

48. My goals are realistic.

49. Failure on a task means I am a failure. (R)

50. I am pessimistic about my future. (R)

51. I take a logical approach to problem solving rather than an emotional approach.

52. Overcoming obstacles helps me grow.

53. I am proactive in tackling challenges.

54. I have overcome setbacks to conquer a challenge. *

55. I persist despite discouragement from others.

56. I come up with creative solutions.

57. Optimism is important for success.

58. I take criticism personally. (R)

59. I persist at long-term goals.

60. Nothing worthwhile is easy.

61. I find silver linings in unfortunate experiences.

62. I avoid dealing with difficult circumstances. (R)

63. My fears holds me back. (R)

64. It is easy to overcome hardship.

65. I am able to laugh at my situation(s).

66. I keep going even when I am unmotivated.

67. I fight for my goals.

68. When people doubt me, it makes me fight harder.

69. Sometimes the best things come out of the most adverse circumstances.

70. Overcoming hardships takes a lot of effort. (R)

\section{Validity Questions}

1. I am resilient.

2. I have good coping strategies.

3. I recover quickly from hard times.

4. I am calm in a crisis.

5. Overcoming obstacles make me stronger.

6. I learn from my mistakes. 
7. Failure is a learning experience.

8. Challenges can be overcome.

9. I persevere despite adversity.

10. I have control over my life.

References

Duckworth, A.L., Peterson, C., Matthews, M.D., \& Kelly, D.R. (2007). Grit: Perseverance and passion for long-term goals. Journal of Personality and Social Psychology, 9, 1087-1101. *

Smith, B. W., Dalen, J., Wiggins, K., Tooley, E., Christopher, P., \& Bernard, J. (2008). The brief resilience scale: assessing the ability to bounce back. International Journal of Behavioral Medicine, 15(3), 194-200. ** 


\section{Appendix B - Final set of resilience items}

Strongly Disagree

1

\author{
Disagree
}

2

\author{
Neutral
}

3

\begin{abstract}
Agree
\end{abstract}
4
Strongly Agree

5

1. I take on challenges even when the outcome is uncertain.

2. I have gotten stronger over time.

3. I face challenges head on.

4. I come back even stronger after challenging situations.

5. Even if I fail now, I can do better in the future.

6. The future is in my control.

7. I handle setbacks well.

8. I am confident in my abilities.

9. I maintain a hopeful outlook.

10. Even in the hardest circumstances, I manage to find positivity.

11. I recover from failures.

12. Overcoming obstacles helps me grow.

13. I am proactive in tackling challenges.

14. I have overcome setbacks to conquer a challenge.

15. I persist at long-term goals.

\section{Validity Questions}

1. I am resilient.

2. I have good coping strategies.

3. I recover quickly from hard times.

4. I am calm in a crisis.

5. Overcoming obstacles make me stronger.

6. I learn from my mistakes.

7. Failure is a learning experience.

8. Challenges can be overcome.

9. I persevere despite adversity.

10. I have control over my life. 


\section{Appendix C - Instructions Given to Participants}

IMPORTANT: The questions you are being asked in this questionnaire are part of a student project for an undergraduate university psychology class on test construction and design. The student who is giving you this questionnaire has formulated these questions as part of a class assignment. S/he has no special qualifications as a psychologist. Your participation in this project is purely voluntary and anonymous. You will not be reimbursed for participating. You are not required to answer any questions at all, and may omit answers to any particular questions you choose to omit. You are not required to let the student know why you did not answer any omitted questions, nor or are you obligated to provide the student with any information that is not asked on the questionnaire. You may discontinue your participation at any time for any reason, and without providing any explanation. If you choose to withdraw after partially completing the questionnaire, you may keep the questionnaire to dispose of yourself. If you complete the questionnaire, it will never be associated with your name. If you filled it out on paper, that paper will be disposed of by the student in its anonymous state, within three months.

The instructor and supervisor of this course is Dr. Chris Westbury. If you have any concerns about this questionnaire or the class project, please contact him at 492-5275, or chrisw@ualberta.ca. If you wish to know more about the course for which this data is being collected, please visit the course website at http://www.ualberta.ca/ chrisw/Psych431

If you do not wish to participate, please inform the student who gave you this questionnaire now. 